\title{
Topical issue on Wetting and Drying: Physics and Pattern Formation
}

\author{
Published online: 29 February 2016 - (c) EDP Sciences / Società Italiana di Fisica / Springer-Verlag 2016
}

Wetting and drying are non-equilibrium processes involved in many everyday phenomena; some widely observed examples include the ring-like spots remaining on dishes that have been left drying, or the enhanced edges in watercolour paintings. Most coatings are made by depositing a volatile liquid that contains dispersed colloidal particles or dissolved macromolecules. The liquid is then evaporated until a dry, possibly porous, film is obtained. In this way, several basic problems occur at the different stages of film deposition and evolution during the drying process. In particular, there can be a disequilibrium in the liquid transport to the evaporation surface, and/or the contact line can generate a strong concentration gradient. At the end of drying, a solid network is formed within the film, and the liquid is confined to pores within the network. Capillary forces at the liquid/air interface exert an enormous stress on this network and may cause it to develop usually undesirable crack patterns. Understanding the broad variety of the observed phenomena is highly relevant to industrial processes ranging from inkjet printing, spray coating, painting and pesticide spreading to materials engineering such as self-assembly via evaporation and preparation of precursors of ceramics. From the fundamental point of view, wetting on a substrate and drying involves the essential physics which are highly desired by the community of Soft Matter. Despite the long history of research in the topic, it is still quite challenging to obtain a full understanding of wetting and drying due to the complexity of the liquid-substrate interaction, the specific behavior of the fluid fields at singularities, the modification of the rheological properties of the system, and the occurrence of hydrodynamic or mechanical instabilities.

This themed issue highlights both experimental and theoretical approaches to our understanding of the underlying physics of wetting dynamics, as well as novel wetting and drying phenomena including pattern formation. Some contributions are devoted to answering to the following questions. How do the thickness and profile of the film evolve in relation to the rheological properties of the film? How does the complex motion of a contact line depend on the surface structure and affect a sessile drop evolution? How does a locally non-homogeneous evaporation process affect the film formation? Questions addressed by these contributions are not only important from a fundamental physics point of view, but also have clear implications for many industrial applications.

This topical issue presents the wetting of different liquids, such as polymeric films by their solvents [1] and by liquid crystals [2]. A contribution is devoted to determining locally the profile of thin films using colour interferometry techniques [3]. The dependence of wetting transition on the hierarchical structured substrate is considered [4], and the electrowetting mechanism for the manipulation of a droplet in a immiscible liquid is investigated [5]. Due to the wetting of micro/nano scaled hydrophobic particles with the inner liquid core, liquid marble exhibits intriguing elastoplasticity and results in excellent sculpture performance [6]. The deposition pattern on a substrate is governed by the different flow regimes, the contact line motion and the diffusion of the containing materials [7-10]. The film formation deals with the drying process, which is influenced by the initial concentration and evaporation rate [11], the geometry of a capillary channel [12], and the roughness of the substrate [13]. The drying mechanisms of porous materials [14] and the pattern formation of colloidal droplets under a mask [15] are also highlighted. We believe the topical collection of these outstanding contributions will stimulate new researches and make progress in the field of wetting and drying, and its pattern formation. 
We are delighted to publish this special issue to our readers and also want to express our sincere thanks to Solange Guéhot in the editorial office.

\section{References}

1. F. Lequeux et al., Eur. Phys. J. E 39, 12 (2016).

2. Nuno M. Silvestre et al., Eur. Phys. J. E 39, 13 (2016).

3. Calum S. Butler et al., Eur. Phys. J. E 39, 14 (2016).

4. Kejun Lin et al., Eur. Phys. J. E 39, 15 (2016).

5. Yanbo Xie et al., Eur. Phys. J. E 39, 16 (2016).

6. Janlin Liu et al., Eur. Phys. J. E 39, 17 (2016).

7. Xin Zhong et al., Eur. Phys. J. E 39, 18 (2016).

8. Mohar Dey et al., Eur. Phys. J. E 39, 19 (2016).

9. Diego Noguera-Marín et al., Eur. Phys. J. E 39, 20 (2016).

10. Y. Msambwa et al., Eur. Phys. J. E 39, 21 (2016).

11. Fanlong Meng et al., Eur. Phys. J. E 39, 22 (2016).

12. Emmanuel Keita et al., Eur. Phys. J. E 39, 23 (2016).

13. Tingting Liu et al., Eur. Phys. J. E 39, 24 (2016).

14. Joel Songok et al., Eur. Phys. J. E 39, 25 (2016).

15. Yuri Yu Tarasevich et al., Eur. Phys. J. E 39, 26 (2016).

Duyang Zang, Ludovic Pauchard, and Wei Shen 